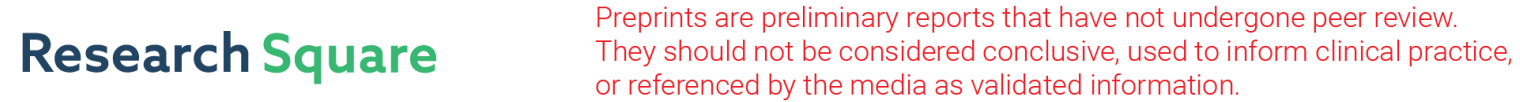 \\ Time to and extent of widespread testing for COVID- 19 outbreak: Experience from the first wave in Australia and Italy
}

Sumaiya Abedin ( $\sim$ su.abedin@gmail.com )

University of Rajshahi

Atikur Khan

Qantares, Australia

Saleheen Khan

Minnesota State University, USA

\section{Research Article}

Keywords: COVID-19, panel generalized linear model, test positivity rate, population density

Posted Date: September 14th, 2020

DOl: https://doi.org/10.21203/rs.3.rs-75767/v1

License: (1) (i) This work is licensed under a Creative Commons Attribution 4.0 International License.

Read Full License 


\title{
Time to and extent of widespread testing for COVID-19 outbreak: Experience from the first wave in Australia and Italy
}

\author{
Sumaiya Abedin ${ }^{1 *}$, Atikur R Khan ${ }^{2}$ \\ and Saleheen Khan ${ }^{3}$
}

${ }^{*}$ Correspondence:

su.abedin@gmail.com

${ }^{1}$ Department of Population

Science, University of Rajshahi, Rajshahi 6205, Bangladesh

Full list of author information is available at the end of the article

\begin{abstract}
Background: At the beginning of COVID-19 outbreak, very little was known about its control options and most of the intervention options relied mainly on other virus outbreak and influenza epidemic. Different countries started responding to this epidemic somewhat in different ways to achieve a common goal of transmission reduction. Population-wide intervention measures such as social distancing, testing and isolation were implemented in different countries. However, commonly adopted intervention measures impacted different countries in different ways. Differential effects of those interventions become apparent in Australia and Italy, where Australia's success to control the epidemic has been in limelight. Differences in time to and extent of widespread testing are likely to have differential effects on the daily number of confirmed cases in both countries.

Methods: We apply panel generalized linear models for daily number of cases to explore differential effects of timing to and extent of widespread testing on daily number of cases. We have analyzed daily number of confirmed cases data from the first reported cases in Australia and Italy to 31 May 2020. Our data sets can be downloded from an open source database at https://ourworldindata.org.

Results: More tests during the early stage of outbreak prior to reach the peak may reduce the daily number of cases by almost $40 \%$. Only $1 \%$ increase in test positivity on the $(t-5)$ th day may incur $1.84 \%$ increase in daily number of cases on the $t$ th day. For $1 \%$ increase in test positivity rate on the $(t-5)$ th day, a country with one unit higher logarithm of population density may result in 2.82 times higher number of cases on the $t$ th day.

Conclusion: Conducting widespread testing during the early stage prior to reaching the peak has favored Australia to control the outbreak much faster than Italy. Early adoption of widespread testing with lower degree of test positivity rates flattens the curve faster. Population density has a moderating effect. Even if the test positivity rate is the same, a region with higher population density is likely to experience a peak with higher number of daily confirmed cases.
\end{abstract}

Keywords: COVID-19; panel generalized linear model; test positivity rate; population density

\section{Background}

As the coronavirus disease 2019 ( COVID-19) alarmed loudly and the World Health Organization (WHO) officially declared a pandemic on 13 March 2020, countries saw little choice to control this epidemic. Italy, the hardest hit European country 
with exceptionally good healthcare service, started to decide about whom to treat and whom to give up. Entire population were on almost complete lockdown with police and military personnel to patrol the street to avoid curfew breaches. Hong Kong, Singapore and South Korea on the other hand emphasised on surveillance, widespread testing to identify and quarantine all positive cases, and tracing contacts of positive cases to test or quarantine them [1,2]. The first COVID-19 case is reported in Australia on 25 January 2020 and the first case in Italy is reported on 31 January 2020. As of 31 May 2020, Australia has 7,185 confirmed cases whereas Italy's healthcare system has been jeopardised with 232,664 confirmed cases. Australia has been a success story in controlling this epidemic. Despite of active implementation of similar interventions, Italy's experience is nothing short of nightmare with almost 33,340 deaths by the end of May 2020. Differential effects of similar interventions and their correlates in two countries have been an interesting domain to researchers. We would like to conduct empirical investigation to explore factors behind such differential effects for suppression of the epidemic.

Suppression of an epidemic can be done by implementing appropriate intervention such as population level social distancing, however, transmission continues once the initial suppression interventions are relaxed. Initial suppression intervention could only be aimed at getting more time to enhance testing and contact tracing to adopt locally targeted response to maintain lower level of transmission once the interventions are relaxed [3]. Thus a combined suppression intervention based on population level social distancing, testing, contact tracing and quarantining is necessary to control this epidemic. WHO Director General Tedros Adhanom Ghebreyesus stressed to adopt a comprehensive approach at a press conference by saying "Not testing alone. Not contact tracing alone. Not quarantine alone. Not social distancing alone. Do it all" [2]. Australia has successfully adopted all these strategies to control the COVID-19 epidemic. In spite of implementation of all these control interventions, COVID-19 hit Italy harder than Australia. Differential approaches in adopting these control interventions may be a source of differential effect of these interventions in two countries.

"Test, test, and test again" has been a known strategy in identifying a case to control HIV epidemic [4]. As a suppression intervention for COVID-19 epidemic, a similar "test, test and test" strategy has been announced by WHO Director General [5]. It is not possible to test whole population at the same time to identify all positive test cases and isolate them to restrain any further transmission. Thus an instant suppression intervention such as population level social distancing may provide some time to prepare for widespread testing by contact tracing. Mathematical models and data-driven decision making is quintessential to depict scenarios in flattening the curve and a persistent test positivity rates below $10 \%$ for weeks is considered to open up businesses again [6]. Each moment during an epidemic costs with more cases and thus create more burden on healthcare systems. Any failure in initial suppression intervention costs more in later stages [3]. Time to adopt any intervention will affect flattening the curve too [7]. Thus time to start widespread testing is an important factor to consider for early control of an outbreak.

Any delay in widespread testing or conducting only a fewer number of tests would allow an index to transmit virus to many people. Similarly, conducting fewer number 
of tests purposively and on priority basis is likely to ignore many asymptomatic cases that in turn results in a prolonged epidemic regime. Thus timing and extent of widespread testing are two key factors that play important roles in containing an outbreak. One major challenge is to quantify the amount of test to term as widespread test for controlling an epidemic outbreak. Our aim in this empirical study is to examine differential impact of these two factors on daily number of COVID-19 episodes.

Social and physical distancing is the most commonly adopted suppression intervention in virus epidemics. In areas with high population density, the distance between individuals is shorter and the spread range of individuals is wider. Because of higher contact frequency and more neighbors around them, infectivity and the probability of being infected also increase with the increase of population density [8-10]. Thus population density is likely to have an effect on the number of daily cases, and cities or regions or countries with higher population density is likely to hit harder by COVID-19 epidemic. Thus we would like to explore any moderating effect of population density to learn more about the distribution of mean daily number of episodes under different settings of widespread testing.

Our contributions to this study are in exploring differential effects of (1) time to conduct widespread testing (2) extent of widespread testing, and (3) population density to examine the distribution of mean number of episodes under widespread testing. We also explore Australia's strategy to control the first wave of epidemic and the similar strategy to flatten the curve faster with fewer number of daily confirmed cases during the second wave of epidemic.

\section{Data and methods}

Data used in this empirical study are collected from open source data services for COVID-19. Daily and cumulative number of cases for both countries can be downloaded from the most popular open source databases at https://ourworldindata. org. Some more data on daily number of tests and test positivity rates for Australia are obtained from https://www.covid19data.com.au and those for Italy can be downloaded from https://github.com/pcm-dpc/COVID-19.

Daily number of confirmed cases is an integer and a count response model is suitable to predict the outcome. Poisson regression is commonly used in analyzing count data in response to other predictor variables. When time series of counts are formed panels with two countries, the underlying data set forms a panel data frame and a panel generalized linear model (PGLM) is used to predict the daily number of confirmed cases in response to other predictor variables. The $\mathrm{R}$ package $\mathrm{pglm}$ is used to fit the model [11] and the Hausman test [12] is used to choose between fixed or random-effects models [13].

\section{Results}

Time-lag relationship

Without any intervention to barrier the outbreak the number of infected individuals would grow exponentially. Let us assume that an infected person is not aware of infection and so does not take care of any protective measure to stop transmitting to others until the hospitalization or isolation. Assuming mean duration between 
onset of symptoms and hospitalization (or isolation) is $\tau$ days, number of episodes on day $t$ is

$$
I_{t}=m \sum_{j=0}^{\tau-1} I_{t-s-j}
$$

where $m$ is the mean daily number of individuals who are supposed to get virion from an infected person and $s$ is the serial interval (difference between the symptom onset time of an infectee and the symptom onset time of infector).

Equation (1) computes the number of transmissions accumulated by the previously infected persons up to $\tau$ days prior to their hospitalization (or isolation). Median incubation period of SARS-CoV-2, the novel coronavirus for COVID-19 disease is 5.1 days [14]. Li et al. [15] have demonstrated that the transmission can happen during the terminal stage of incubation period. The serial interval (time from illness onset from an infector to illness onset of an infectee) of COVID-19 has been estimated to be close to or shorter than the median incubation period [16]. In their review work, Liu et al. [17] explored that the the transmissibility of a virus (the average number of new infections generated by an infectious person) can vary greatly depending on the mathematical models and assumptions placed in estimating this. Statistical model based estimates vary between 2.2 to 3.58 with an average of 2.67 days $[15,17,18]$. With higher number of contacts such as festival seasons, as in Wuhan prior to Spring Festival, this could reach up to 6.47 with $95 \%$ confidence interval 5.71-7.23 [19].

Equation (1) can also be expressed as a function of $\tau$-day rolling mean

$$
I_{t}=\beta\left(\frac{\sum_{j=0}^{\tau-1} I_{t-s-j}}{\tau}\right)=\beta \bar{I}_{t-s}(\tau)=f\left(\bar{I}_{t-s}(\tau)\right),
$$

where the constant $\beta=m \tau$ is unknown in practice and $\bar{I}_{t-s}(\tau)$ is the $\tau$-day average of daily reported cases. In practice, an infected person will not transmit virion to equal number of individuals each day until hospitalization or isolation or recovery. Thus the coefficient $\beta$ represent an average number of persons infected by an infected individual in $\tau$ days after the serial interval of $s$ days. Since $I_{t}$ is the daily count of COVID-19 cases, a Poisson model is suitable to explain the relationship between $I_{t}$ and $\bar{I}_{t-s}(\tau)$. Thus we apply Poisson generalized linear model (PGLM) to daily number of COVID-19 cases in Australia and Italy. We consider 120 days data since the first reported case in Australia (25 January to 31 May 2020) and Italy (31 January to 31 May 2020) shown in Figure 1.

To explore the effect of $\tau$ on daily number of COVID-19 cases, we apply Poisson regression of $I_{t}$ on $\bar{I}_{t-5}(\tau)$ for $\tau=1,2, \ldots, 10$ and plot corresponding Akaike Information Criterion (AIC) in Figure 2. The minimum AIC is achieved for $\tau=2$ for Australian data set and that is minimum at $\tau=3$ for Italian data set.

We are not going to explore the best model for prediction of daily number of cases, rather our focus is on to explore the significance of $\bar{I}_{t-5}(\tau)$. Since $\bar{I}_{t-s}(2)=$ $\sum_{j=0}^{2-1} I_{t-s-j} / 2$, Australian COVID-19 patients are likely to have spent 2 days prior to test and isolation or hospitalization. On the other hand, Italian patients are likely 
(a) Australia

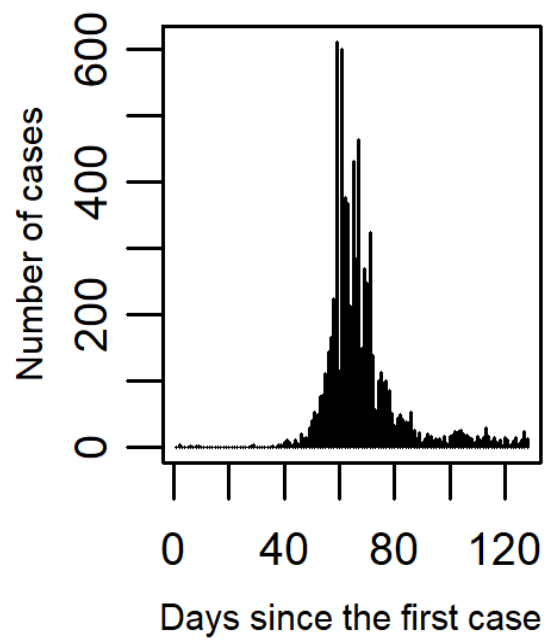

(b) Italy

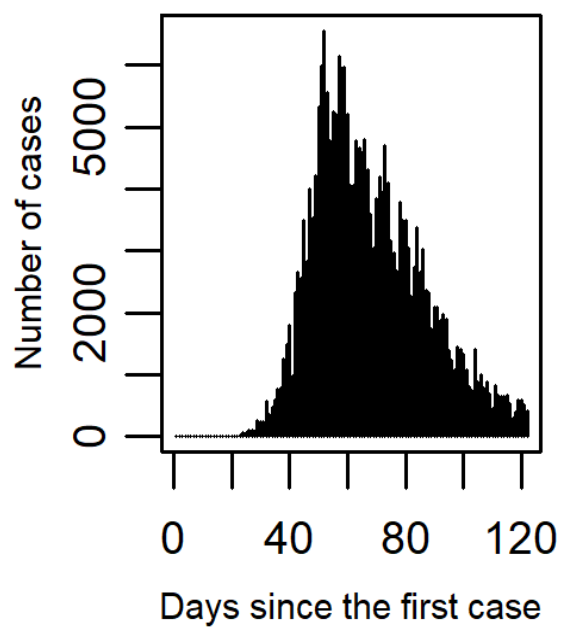

Figure 1 Daily number of COVID-19 cases from the first detection to 31 May 2020 in (a) Australia and (b) Italy.

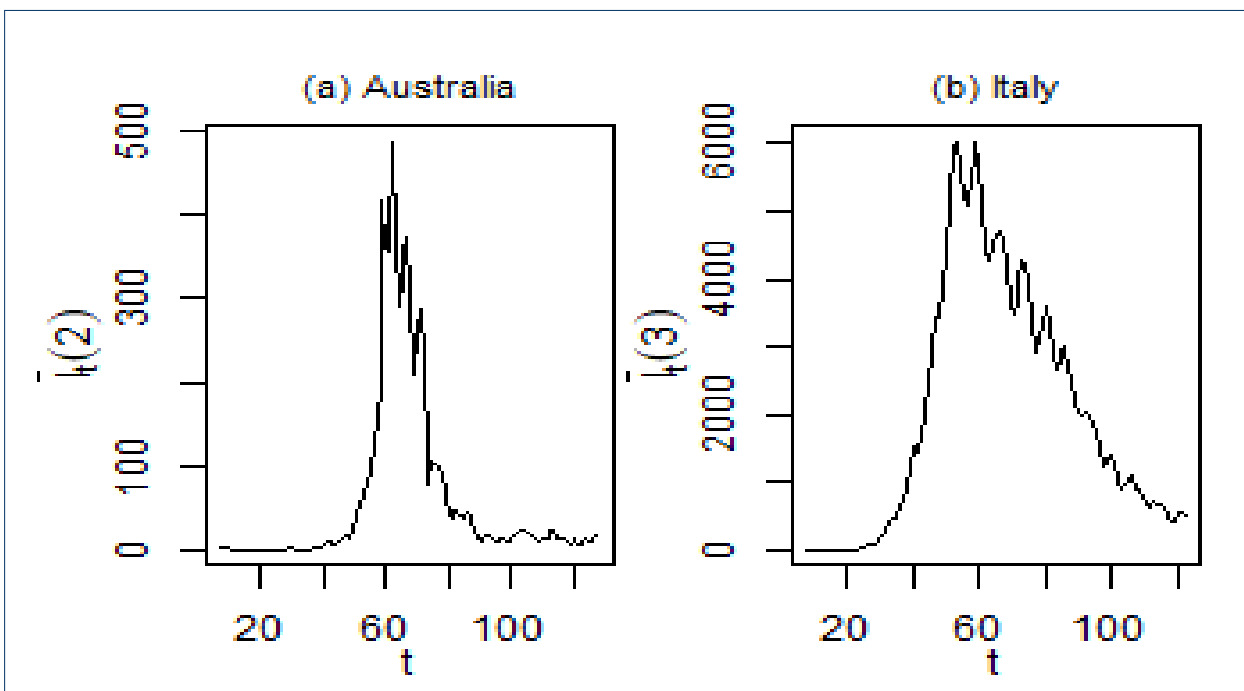

Figure 2 Akaike information criterion (AIC) for different values of $\tau=1,2, \ldots, 10$.

to have spent 3 days prior to test and isolation or hospitalization. This result reveals that the COVID-19 patients might have been identified a day earlier in Australia than in Italy.

Figure 1 shows a pattern that the number of daily cases increases at the beginning, reaches to its peak and then declines. This pattern can be explained by an inclusion of quadratic form of trend in the Poisson model. Results of fitted Poisson models shown in Table 1 support that the number of cases on the $t$ th day in Australia is a 
function of 2-day rolling mean with 5-day lag and quadratic trend. Significance of coefficients divulges that the number of cases on the $(t-5)$ th and $(t-6)$ th days contribute significantly to the number of cases on the $t$ th day in Australia.

Table 1 Effect of $\tau$ on daily number of cases in a Poisson model.

\begin{tabular}{l|ccc|ccc}
\hline \multirow{2}{*}{ Model Term } & \multicolumn{3}{|c|}{ Australia, $\tau=2$} & \multicolumn{3}{c}{ Italy, $\tau=3$} \\
\cline { 2 - 7 } & $\hat{\beta}$ & $\exp (\hat{\beta})$ & $z$-value & $\hat{\beta}$ & $\exp (\hat{\beta})$ & $z$-value \\
\hline Intercept & -3.2230 & 0.0398 & -17.2900 & 2.8630 & 17.5140 & 111.4000 \\
$\bar{I}_{t-5}(\tau)$ & 0.0025 & 1.0025 & 26.4700 & 0.0001 & 1.0001 & 34.1500 \\
$t$ & 0.2356 & 1.2657 & 40.4200 & 0.1566 & 1.1695 & 163.5000 \\
$t^{2}$ & -0.0017 & 0.9983 & -41.3500 & -0.0011 & 0.9989 & -166.7800 \\
\hline
\end{tabular}

A similar exposition holds for Italian epidemic outbreak with the number of cases as a function of 3-day rolling mean with 5-day lag and quadratic trend. Number of daily episodes on the $(t-5) \mathrm{th},(t-6) \mathrm{th}$, and $(t-7)$ th days contribute significantly to the number of episodes on the $t$ th day in Italy. Thus the Poisson models in Table 1 also support the exposition that the Australian COVID-19 patients might have been identified earlier than Italian patients.

\section{Early widespread testing}

As has been explored in the previous section, the main reason behind identifying Australian patients earlier than their Italian counterpart might be an effect of conducting higher number of tests in Australia. Figure 1 and Figure 3 show that the peak in Australia is observed on the 59th day (in the $9 t h$ week) from the first case and that in Italy is observed in late March 2020 on the 52nd day (in the 8th week) from the first reported case [20]. A comparative peak epidemic scenarios in Australia and Italy shown in Table 2. Prior to reaching the peak, Australia has conducted almost 5.608 tests per thousand and Italy has conducted 4.274 tests per thousand of population. Thus more tests in Australia might have helped to identify infected persons earlier than in Italy to reduce the spread of virion from an infector to an infectee.

Table 2 Peak epidemic scenarios in Australia and Italy: Key statistics on the day before and after the day with the highest number of daily incidences.

\begin{tabular}{l|ccc|ccc}
\hline \multirow{3}{*}{ Key Statistics } & \multicolumn{3}{|c|}{ Australia } & \multicolumn{3}{c}{ Italy } \\
\cline { 2 - 7 } & $t=58$ & $t=59$ & $t=60$ & $t=51$ & $t=52$ & $t=53$ \\
& $22-$ Mar-2020 & $23-$ Mar-2020 & 24-Mar-2020 & 21-Mar-2020 & 22-Mar-2020 & 23 -Mar-2020 \\
\hline Total cases per million & 43.0590 & 67.0200 & 71.4910 & 777.6970 & 886.1460 & 978.1050 \\
New cases per million & 8.7840 & 23.9610 & 4.4710 & 99.0050 & 108.4490 & 91.9590 \\
Total death per million & 0.2750 & 0.2750 & 0.2750 & 66.6870 & 79.8350 & 90.5700 \\
New death per million & 0 & 0 & 0 & 10.3370 & 13.1490 & 10.7340 \\
Total test per thousand & 4.9800 & 5.6080 & 6.2750 & 3.8570 & 4.2740 & 4.5560 \\
New test per thousand & 0.4710 & 0.6270 & 0.6670 & 0.4360 & 0.4160 & 0.2820 \\
Test positivity (in \%) & 1.8700 & 3.8200 & 0.6700 & 22.7300 & 26.0400 & 32.5800 \\
\hline
\end{tabular}

Here, $t$ is the number of days since the first reported incidence.

We examine the effect of conducting higher number of tests in early stages prior to reaching the peak by using a dummy variable in panel GLM. The dummy variable assumes a value of 1 if number of tests in one country exceeds the other. Since Australia conducts higher number of tests prior to reaching the peak point of the outbreak, it assumes 1 for the first 59 days for Australia in the panel data frame. Estimated coefficients of fixed effect panel GLM are shown in Table 3. 

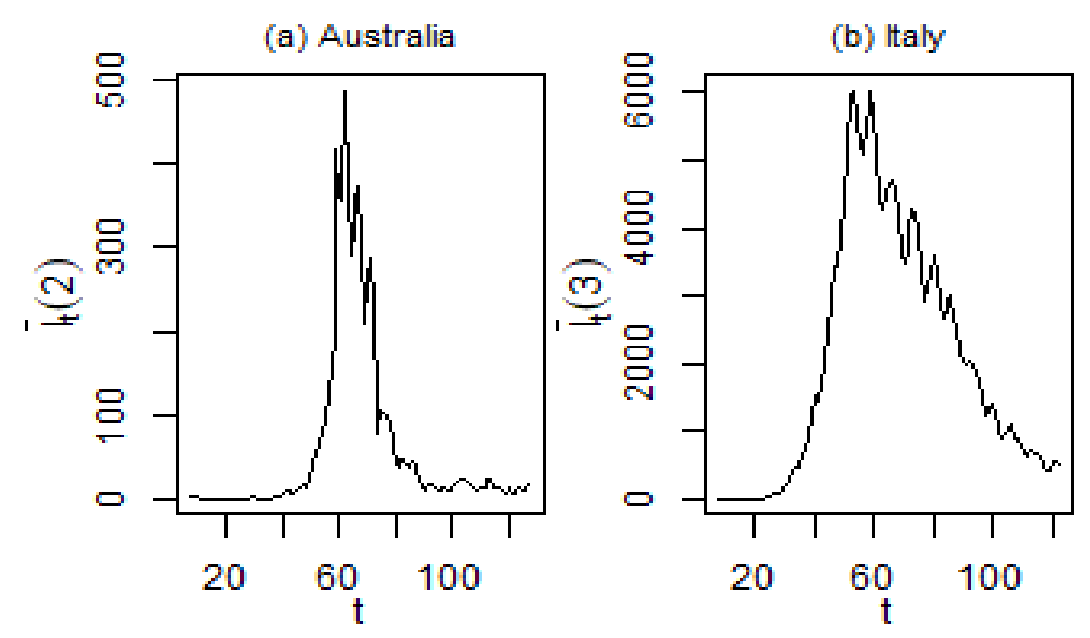

Figure 3 Rolling mean $\bar{I}_{t}(\tau)=\sum_{j=0}^{\tau-1} I_{t-j} / \tau$ where $t$ is the number of days since the first case.

Table 3 Effect of conducting more tests prior to reach the peak in a fixed effect panel GLM.

\begin{tabular}{lccc}
\hline Model Term & $\hat{\beta}$ & $\operatorname{Exp}(\hat{\beta})$ & $t$-stat \\
\hline$I_{t-5}(3)$ & 0.0001 & 1.0001 & 28.9000 \\
$t$ & 0.1636 & 1.1777 & 174.3000 \\
$t^{2}$ & -0.0012 & 0.9988 & -178.3000 \\
More tests before peak & -0.5105 & 0.6002 & -18.2000 \\
\hline \multicolumn{4}{l}{ All coefficients are significant at 5\% level. }
\end{tabular}

Coefficient of number of days since the first incidence $(t)$ has positive sign whereas the coefficient of $t^{2}$ is negative. Thus the number of days since the first incidence has quadratic effect: Number of daily cases increase with the number of days, reaches to the peak and declines thereafter. It mimics that the model clearly explores the nature of an epidemic outbreak. Australia has conducted higher number of tests per thousand of population prior to reach the peak of this epidemic and the effect is found significant with a negative coefficient. It seems that more tests during the early stage of outbreak may reduce the episodes rate by almost $40 \%$. Thus higher number of tests during the early stages of outbreak prior to reach the peak has helped Australia to contain the outbreak much better than Italy.

\section{Test positivity rates}

Number of tests required for widespread testing may depend on the type and extent of transmission. Test positivity rate (number of positive cases as a percent of total number of tests) is an indicator to test sufficient number of suspected contacts to a COVID-19 patient. A test positivity rate below $10 \%$ for two consecutive weeks has been recommended by the Centers for Disease Control and Prevention(CDC) for transition from phase-2 to phase-3 [6]. Test positivity rate over $10 \%$ is considered as "warning flag". In a media briefing on 31 March 2020, the WHO mentioned that the test positivity rate over $10 \%$ is alarming in the sense that many of the cases will be missing if the positivity rate is very high. Of course, this rate may vary depending on 
the availability of test kits and capability of testing against the extent of community transmission (https://twitter.com/WHO/status/1244637360706342917).

We find that the five-day lag in widespread testing $\left(r_{t-5}<10 \%\right)$ is highly significant in a fixed effect panel GLM (Table 4). On average, test positivity rate less than $10 \%$ on the $(t-5)$ th day causes almost $26 \%$ reduction in number of COVID-19 episodes on the $t$ th day compared to the test positivity rate greater than or equal to $10 \%$. Australia has maintained a very low test positivity rates from the beginning of the epidemic and this might have enabled Australia to successfully contain the outbreak.

Table 4 Estimates of parameters in a fixed effect panel GLM of daily episodes in response to test positivity rates in Australia and Italy.

\begin{tabular}{lccc}
\hline Model Term & $\hat{\beta}$ & $\operatorname{Exp}(\hat{\beta})$ & $t$-stat \\
\hline$I_{t-5}(3)$ & 0.0001 & 1.0001 & 35.7900 \\
$t$ & 0.0975 & 1.1024 & 83.1600 \\
$t^{2}$ & -0.0007 & 0.9993 & -92.1600 \\
$r_{t-5}<10 \%$ & -0.3055 & 0.7367 & -43.5700 \\
\hline \multicolumn{4}{l}{ Here, $r_{t}$ is the test positivity rate on the $t$ th day and } \\
all coefficients are significant at $5 \%$ level.
\end{tabular}

Population density and test positivity rates

Social distancing reduces the number of contacts within the limited distance and thus reduces the number of new cases substantially to contain the outbreak. People may find it difficult to maintain safe physical distance in highly populated cities and service areas such as shopping centers and other service stations. Thus there might have an impact of population density in the intensity of outbreak. Number of tests and thus the test positivity rate is an independent variable, and population density does not depend on it. At the time of obtaining the test positivity rate, population density is already known. Thus population density is likely to moderate the test positivity. Statistical significance test for interaction term of population density and test positivity can be used to examine the existence of moderating effect [21].

Before going to examine the significance of the interaction between population density and test positivity, we examine the effect of test positivity on daily number of COVID-19 episodes. Results obtained from a panel GLM in Table 5 demonstrates the highly significance of test positivity on the number of daily episodes. It depicts that $1 \%$ increase in test positivity on the $(t-5)$ th day incurs $1.84 \%$ increase in daily incidences on the $t$ th day after adjusting the effect of other predictors in the model.

Table 5 Pooling estimates in a panel GLM to examine the effect of test positivity in Australia and Italy

\begin{tabular}{lccc}
\hline Model Term & $\hat{\beta}$ & $\operatorname{Exp}(\hat{\beta})$ & $t$-stat \\
\hline Intercept & 6.9310 & 1023.517 & 210.8310 \\
$\bar{I}_{t-5}(3)$ & 0.0004 & 1.0004 & 203.7240 \\
$t$ & -0.0032 & 0.9968 & -3.5920 \\
$t^{2}$ & -0.0007 & 0.9993 & -12.3670 \\
$r_{t-5}$ & 0.0182 & 1.0184 & 46.5630 \\
\hline \multicolumn{4}{l}{ All coefficients are significant at 5\% level. }
\end{tabular}

When we include population density and its interaction term with the test positivity in the panel GLM, test positivity with 5-day lag, population density and 
their interaction terms are found statistically significant at $5 \%$ level (Table 6 ). It can be shown that for $1 \%$ increase in test positivity on the $(t-5)$ th day and a one unit increase in $\log$ (Population Density) may result in $e^{0.2796+0.8060-0.0471}=2.8250$ times higher number of episodes on the $t$ th day.

Table 6 Pooling estimates in a panel GLM to examine the effect of population density in Australia and Italy

\begin{tabular}{lccc}
\hline Model Term & $\hat{\beta}$ & $\operatorname{Exp}(\hat{\beta})$ & $t$-stat \\
\hline Intercept & -0.6850 & 0.5041 & -12.6800 \\
$\bar{I}_{t-5}(3)$ & 0.0001 & 1.0001 & 20.7400 \\
$t$ & 0.1183 & 1.1256 & 93.4100 \\
$t^{2}$ & -0.0008 & 0.9992 & -99.8500 \\
$r_{t-5}$ & 0.2796 & 1.3226 & 32.4400 \\
$\log ($ Density $)$ & 0.8060 & 2.2389 & 150.30 \\
$r_{t-5} \times \log ($ Density $)$ & -0.0471 & 0.9540 & -29.1400 \\
\hline \multicolumn{4}{l}{ All coefficients are significant at $5 \%$ level. }
\end{tabular}

To depict the moderating effect of $\log$ (Density), the logarithmic of population density, we compute expected number of daily COVID-19 cases by setting different values of test positivity rates and population densities. Figure 4 shows distribution of mean number of daily episodes for different test positivity rates and population densities. Even for a similar test positivity rate, higher population density incurs higher number of mean daily cases.

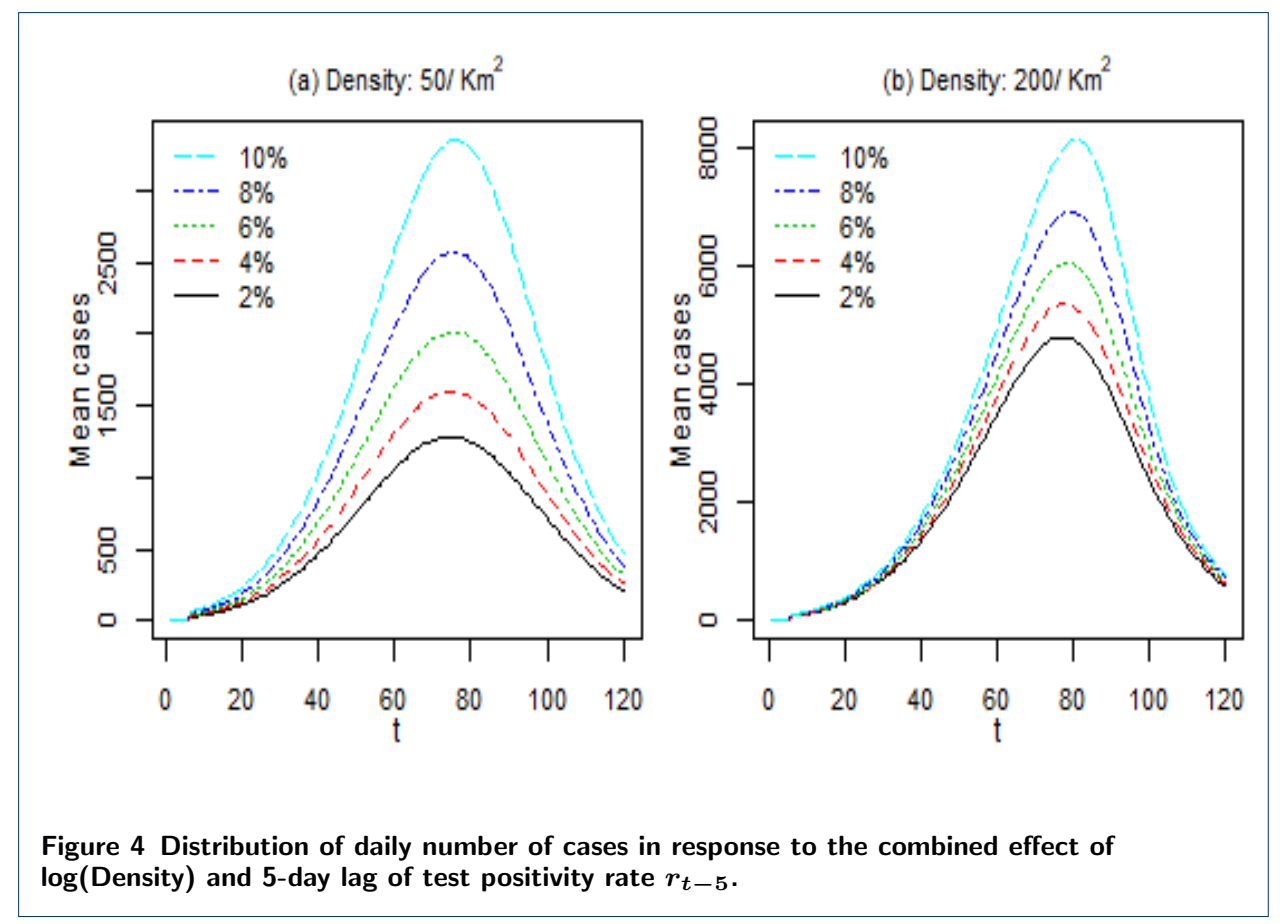

Though a persistent test positivity rate below $10 \%$ is considered reasonably good to control the epidemic, distribution of mean number of cases in Figure 4 suggests lowering the test positivity rate by conducting much higher number of tests to flatten the curve further. When we compare the distribution of mean number of cases for two regions with population densities $50 / \mathrm{Km}^{2}$ and $200 / \mathrm{Km}^{2}$, the peak scenarios for similar level of test positivity rate are different. Higher population 
density seems to trigger a peak of the epidemic with several fold higher number of daily cases. Thus a generalization of test positivity rate below $10 \%$ is not necessarily be good choice for all regions, rather a much lower test positivity rate would be a preferred choice for over populated countries.

From the first wave to the second wave

Both Australia and Italy have passed through the first wave of the epidemic. The second wave has also been observed in Australia with a declining trend from the first week of August 2020. Figure 5 clearly depicts flattening the curve in the second wave of epidemic with a daily maximum of 721 cases.

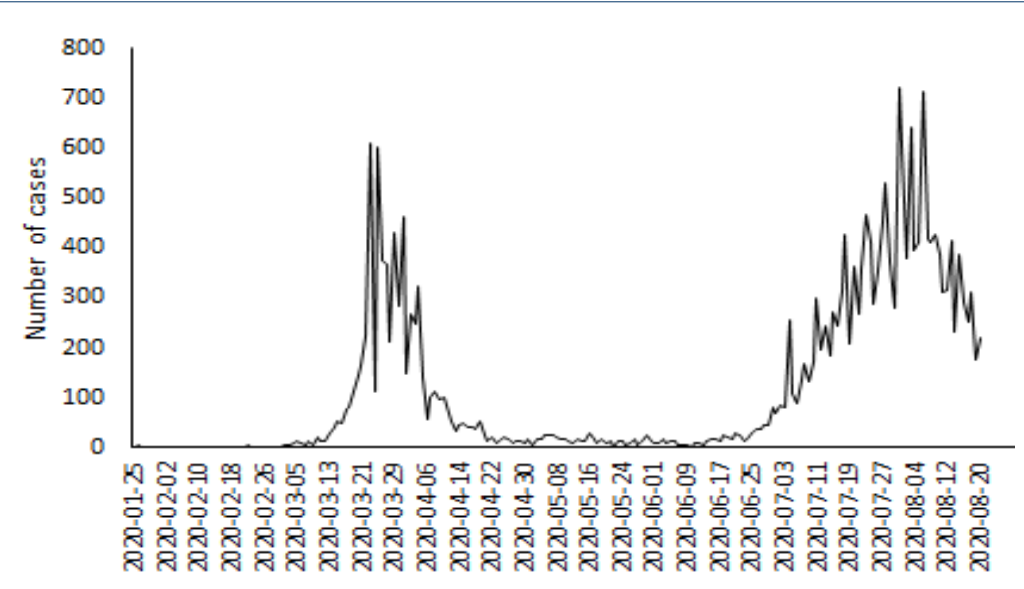

Figure 5 Distribution of daily number of cases during the first and second waves in Australia.

Akin to the control option during the first wave of COVID-19 outbreak, Australia has considered all sort of measures including social and physical distancing, travel restriction and border closure, isolation and quarantine, and widespread testing. Moreover, Australia has adopted the most important strategy to conduct large number of tests so that the test positivity rates become very low. Since the beginning of the second wave, Australia has been conducting widespread testing with test positivity rate less than $1 \%$. Australia is known for very low population density country and results in Figure 4(a) demonstrates that a test positivity rate less than $1 \%$ will provide daily peak cases much lower than 1,000. Thus the results in Figure 4 derived from the first wave of the epidemic are also reflected in the second wave of the epidemic.

\section{Discussion}

Application of similar intervention to different population may affect differently and can vary greatly depending on population distribution. Though similar interventions have been applied in Australia and Italy, success story of Australia in controlling COVID-19 epidemic has been in limelight whereas Italy's story is on the other pole.

Our empirical results demonstrate that the differential effects are mainly due to the higher number of tests conducted in Australia than in Italy during the early stage of the epidemic. More tests during the early stage prior to peak of the epidemic 
reduces the daily number of episodes by almost $40 \%$. Early testing has benefited other countries too. South Korea started widespread testing at the very beginning of the epidemic with the policy of open public testing even for asymptomatic people besides contact tracing. Once the peak is over and the number of new cases falls, South Korea has continued testing with almost similar rate as before. Singapore and Hong Kong also adopted the policy of widespread testing for suppression of transmission at the very beginning of the outbreak. It seems that Italy's policy in contract tracing with lower number of tests has resulted in many missing asymptomatic cases and even might have delayed isolation of symptomatic cases. On the other hand, Australia's success in controlling the epidemic is more inline with the widespread testing in Hong Kong, Singapore, and South Korea to pass any asymptomatic case through the testing net.

Extent of widespread testing has played an important role in containing the virus outbreak. We have found that increase in test positivity rate results in increase of 5 days ahead daily episodes. Lower degree of test positivity rate in South Korea's widespread testing has enabled to contain the outbreak faster than many countries. Test positivity rate in South Korea has always been less than $10 \%$ and even around the peak of the epidemic this was around $6 \%$. Australia has been even more stringent in widespread testing with less than $2 \%$ test positivity rate around the peak. Lower degree of test positivity rate around the peak has resulted in faster decline in daily number of episodes in Australia. Italy, on the other hand, had a record of more than $25 \%$ test positivity rate around the peak of the epidemic, and so the number of cases did not fall quickly even once reach the peak.

Population density moderates the effect of test positivity rate, and $1 \%$ increase in test positivity rate with a one unit increase in log population density may cause 2.82 times higher number for 5 days ahead daily episodes. Reduction in test positivity rate reduces the peak number of episodes and flattens the curve faster. Because of very low test positivity rates and very low population density, number of daily episodes fall down very quickly like avalanche in Australia. Whereas higher population density and higher level of test positivity rates have resulted higher number of daily episodes with longer epidemic regime in Italy. South Korea is one of the most densely populated developed country in the world and is also one of the most successful country in controlling the COVID-19 outbreak. Implementation of early widespread testing with low test positivity rate might be the key factor behind South Korea's success. Though the population density in Italy is much lower than that of South Korea, Italy has failed to avail of this added benefit by conducting higher number of tests in early stages of this epidemic. It seems that a test positivity rate in between those of Australia and South Korea could have been a benchmark for Italy to flatten the curve further with much lower number of episodes even during the peak.

Our findings from this study can be generalized to other countries with a clear message to conduct higher number of tests with low test positivity rate from the very beginning of an outbreak. A test positivity rate below $10 \%$ should not be considered as a gold standard for all regions. Over populated countries should consider a test positivity rate much lower than 10\%, preferably around the rates in Australia. Countries passed through the first wave of this epidemic may also implement 
Australia's strategy to conduct widespread testing with low test positivity rates to control any further wave successfully.

\section{Conclusion}

Widespread testing has been a commonly adopted approach in identifying COVID19 positive cases. Any delay in identifying a positive case is likely to generate more cases. Widespread testing at an early stage of epidemic with high volume of tests lowers the test positivity rates and flattens the curve faster. Unless test positivity rates are kept very low from early stages of the epidemic, over populated countries are likely to experience a peak with higher number of daily confirmed cases than the low population density countries.

\section{Declaration}

Ethics approval and consent to participate

The author did not require ethical approval for this study as the data used in this paper are available online.

Consent for publication

Not applicable.

Availability of data and material

Data used in this study can be obtained mainly from the open source databases that is available at

https: //ourworldindata.org.

Competing interests

The authors declare that they have no competing interests.

Funding

None to report.

Authors' contributions

ARK and SK generated the project idea. ARK and SA carried out data analysis and drafted the manuscript. All authors contributed to further drafts and approved the final manuscript.

\section{Author details}

${ }^{1}$ Department of Population Science, University of Rajshahi, Rajshahi 6205, Bangladesh. ${ }^{2}$ Qantares, WA 6009,

Perth, Australia. ${ }^{3}$ Department of Economics, Minnesota State University, MN 56001, Mankato, USA.

References

1. Koo, J.R., Cook, A.R., Park, M., Sun, Y., Sun, H., Lim, J.T., Tam, C., Dickens, B.L.: Interventions to mitigate early spread of SARS-CoV-2 in Singapore: a modelling study. The Lancet Infectious Diseases 20(6), 678-688 (2020). doi:10.1016/S1473-3099(20)30162-6

2. Cohen, J., Kupferschmidt, K.: Countries test tactics in 'war' against COVID-19. Science 367(6484), 1287-1288 (2020). doi:10.1126/science.367.6484.1287

3. Walker, P.G., Whittaker, C., Watson, O.J., Baguelin, M., Winskill, P., Hamlet, A., Djafaara, B.A., Cucunubá, Z., Mesa, D.O., Green, W., et al.: The impact of COVID-19 and strategies for mitigation and suppression in low-and middle-income countries. Science 369(6502), 413-422 (2020). doi:10.1126/science.abc0035

4. Nardone, A., Delpech, V., Gill, O.N., Fenton, K.A., Anderson, J.: Hiv in the uk: test, test, and test again. Lancet 382(9906), 1687-1688 (2013). doi:10.1016/S0140-6736(13)62377-6

5. Umaru, F.A.: Scaling up testing for COVID-19 in Africa: Responding to the pandemic in ways that strengthen health systems. African Journal of Laboratory Medicine 9(1), 1-2 (2020). doi:10.4102/ajlm.v9i1.1244

6. CDC: CDC activities and initiatives supporting the COVID-19 response and the president's plan for opening America up again. Centers for Disease Control and Prevention, US Department of Health and Human Services (2020). https://www.cdc.gov/coronavirus/2019-ncov/downloads/php/

CDC-Activities-Initiatives-for-COVID-19-Response.pdf

7. Giordano, G., Blanchini, F., Bruno, R., Colaneri, P., Di Filippo, A., Di Matteo, A., Colaneri, M.: Modelling the COVID-19 epidemic and implementation of population-wide interventions in Italy. Nature Medicine 4(5), 397-404 (2020). doi:10.1038/s41591-020-0883-7

8. Bin, S., Sun, G., Chen, C.-C.: Spread of infectious disease modeling and analysis of different factors on spread of infectious disease based on cellular automata. International Journal of Environmental Research and Public Health 16(23), 4683 (2019). doi:10.3390/ijerph16234683

9. Chowdhury, R., Luhar, S., Khan, N., Choudhury, S.R., Matin, I., Franco, O.H.: Long-term strategies to control COVID-19 in low and middle-income countries: an options overview of community-based, non-pharmacological interventions. European Journal of Epidemiology, 1 (2020). doi:10.1007/s10654-020-00660-1 
10. Banik, R., Rahman, M., Hossain, M.M., Sikder, M.T., Gozal, D.: COVID-19 pandemic and Rohingya refugees in Bangladesh: What are the major concerns? Global Public Health (2020). doi:10.1080/17441692.2020.1812103

11. Croissant, Y.: pglm: Panel generalized linear models, R package version 0.2-1 (2017). https://cran.r-project.org/web/packages/pglm/index.html

12. Hausman, J.A.: Specification tests in econometrics. Econometrica: Journal of the Econometric Society, 1251-1271 (1978)

13. Eldomiaty, T., Hammam, R., El Bakry, R.: Institutional determinants of financial inclusion: evidence from world economies. International Journal of Development Issues 19(2), 217-228 (2020). doi:10.1108/IJDI-08-2019-0147

14. Lauer, S.A., Grantz, K.H., Bi, Q., Jones, F.K., Zheng, Q., Meredith, H.R., Azman, A.S., Reich, N.G., Lessler, J.: The incubation period of coronavirus disease 2019 (COVID-19) from publicly reported confirmed cases: estimation and application. Annals of Internal Medicine 172(9), 577-582 (2020). doi:10.7326/M20-0504

15. Li, Q., Guan, X., Wu, P., Wang, X., Zhou, L., Tong, Y., Ren, R., Leung, K.S., Lau, E.H., Wong, J.Y., et al.: Early transmission dynamics in Wuhan, China, of novel coronavirus-infected pneumonia. New England Journal of Medicine 382(13), 1199-1207 (2020). doi:10.1056/NEJMoa2001316

16. Nishiura, H., Linton, N.M., Akhmetzhanov, A.R.: Serial interval of novel coronavirus (COVID-19) infections. International Journal of Infectious Diseases 93, 284-286 (2020). doi:10.1016/j.ijid.2020.02.060

17. Liu, Y., Gayle, A.A., Wilder-Smith, A., Rocklöv, J.: The reproductive number of COVID-19 is higher compared to SARS coronavirus. Journal of Travel Medicine 27(2) (2020). doi:10.1093/jtm/taaa021

18. Zhao, S., Lin, Q., Ran, J., Musa, S.S., Yang, G., Wang, W., Lou, Y., Gao, D., Yang, L., He, D., et al.: Preliminary estimation of the basic reproduction number of novel coronavirus (2019-ncov) in china, from 2019 to 2020: A data-driven analysis in the early phase of the outbreak. International Journal of Infectious Diseases 92, 214-217 (2020). doi:10.1016/j.ijid.2020.01.050

19. Tang, B., Wang, X., Li, Q., Bragazzi, N.L., Tang, S., Xiao, Y., Wu, J.: Estimation of the transmission risk of the 2019-nCoV and its implication for public health interventions. Journal of Clinical Medicine 9(2), 462 (2020). doi:10.3390/jcm9020462

20. Sebastiani, G., Massa, M., Riboli, E.: Covid-19 epidemic in italy: evolution, projections and impact of government measures. European Journal of Epidemiology 35(4), 341 (2020). doi:10.1007/s10654-020-00631-6

21. McClelland, G.H., Judd, C.M.: Statistical difficulties of detecting interactions and moderator effects. Psychological bulletin 114(2), 376 (1993) 
Figures

(a) Australia

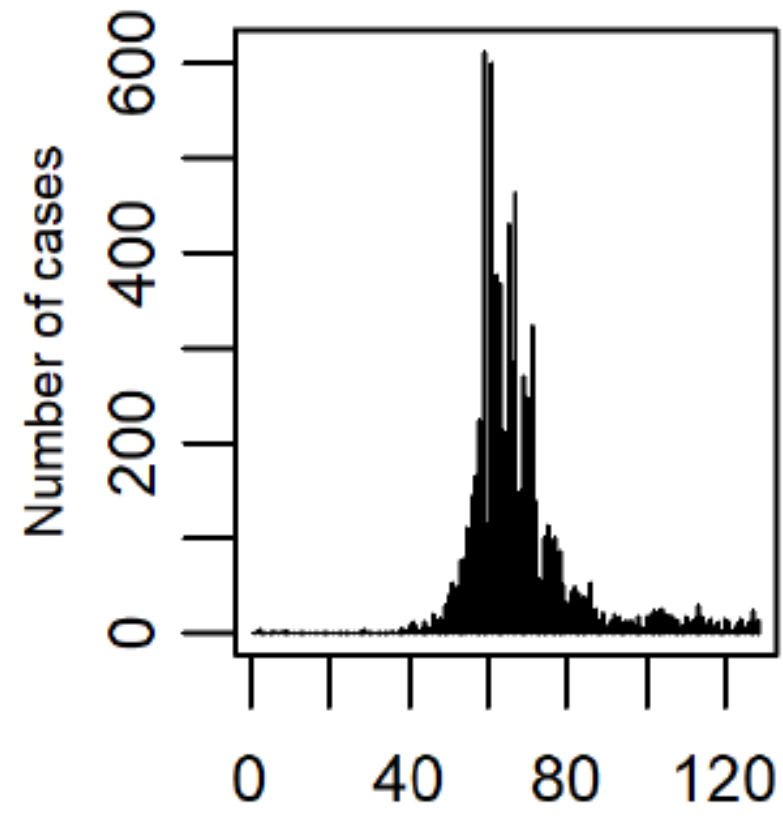

Days since the first case (b) Italy

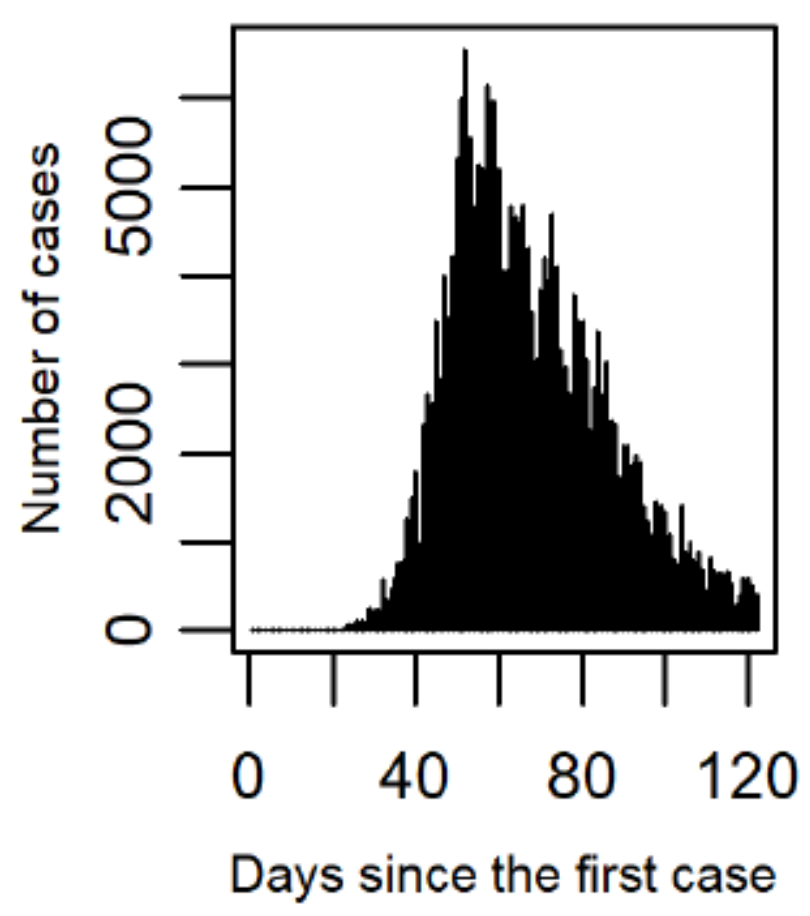

Figure 1

Daily number of COVID-19 cases from the first detection to 31 May 2020 in (a) Australia and (b) Italy. 

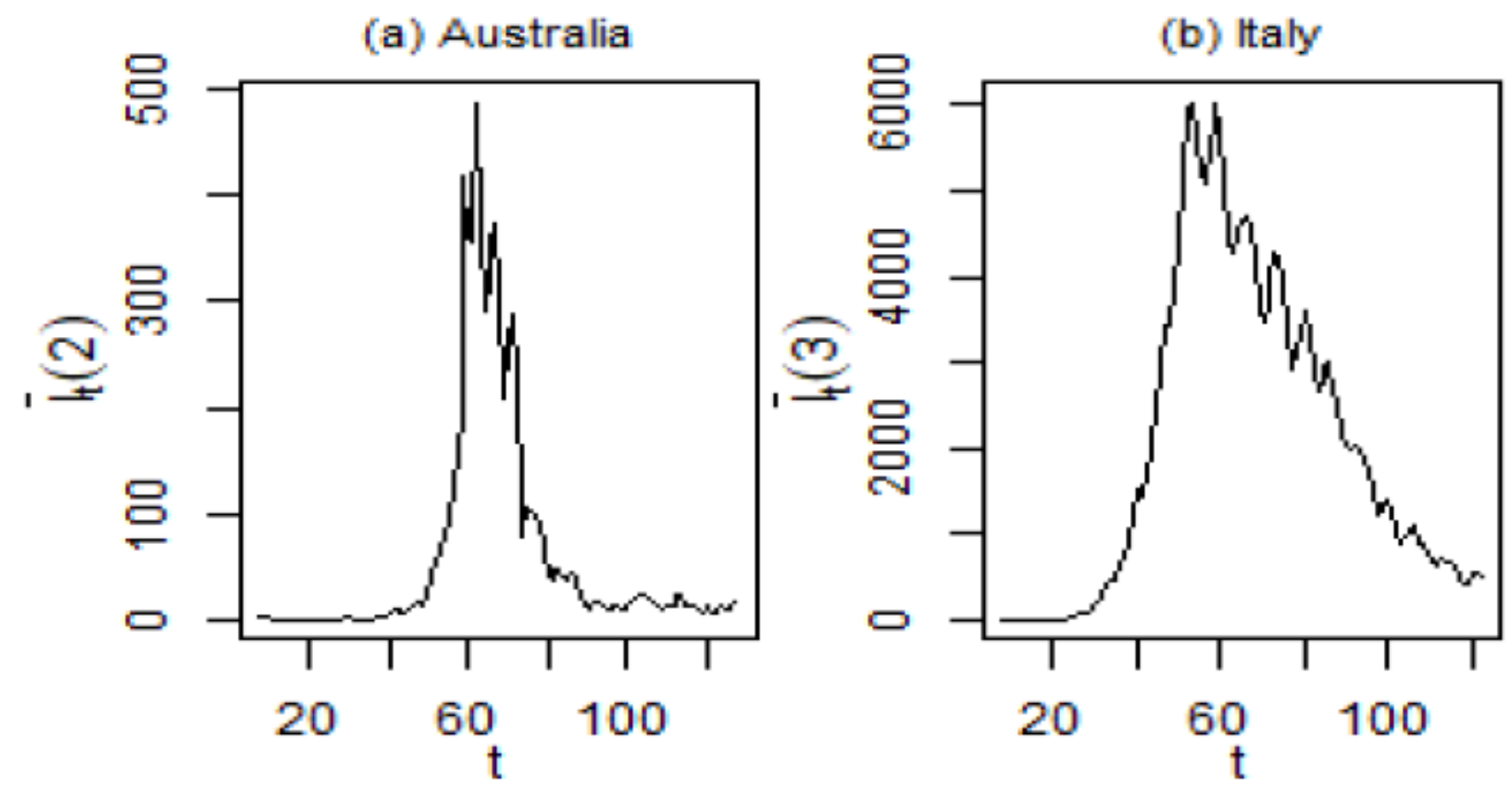

Figure 2

Akaike information criterion (AIC) for different values of $\tau=1,2, \ldots, 10$.
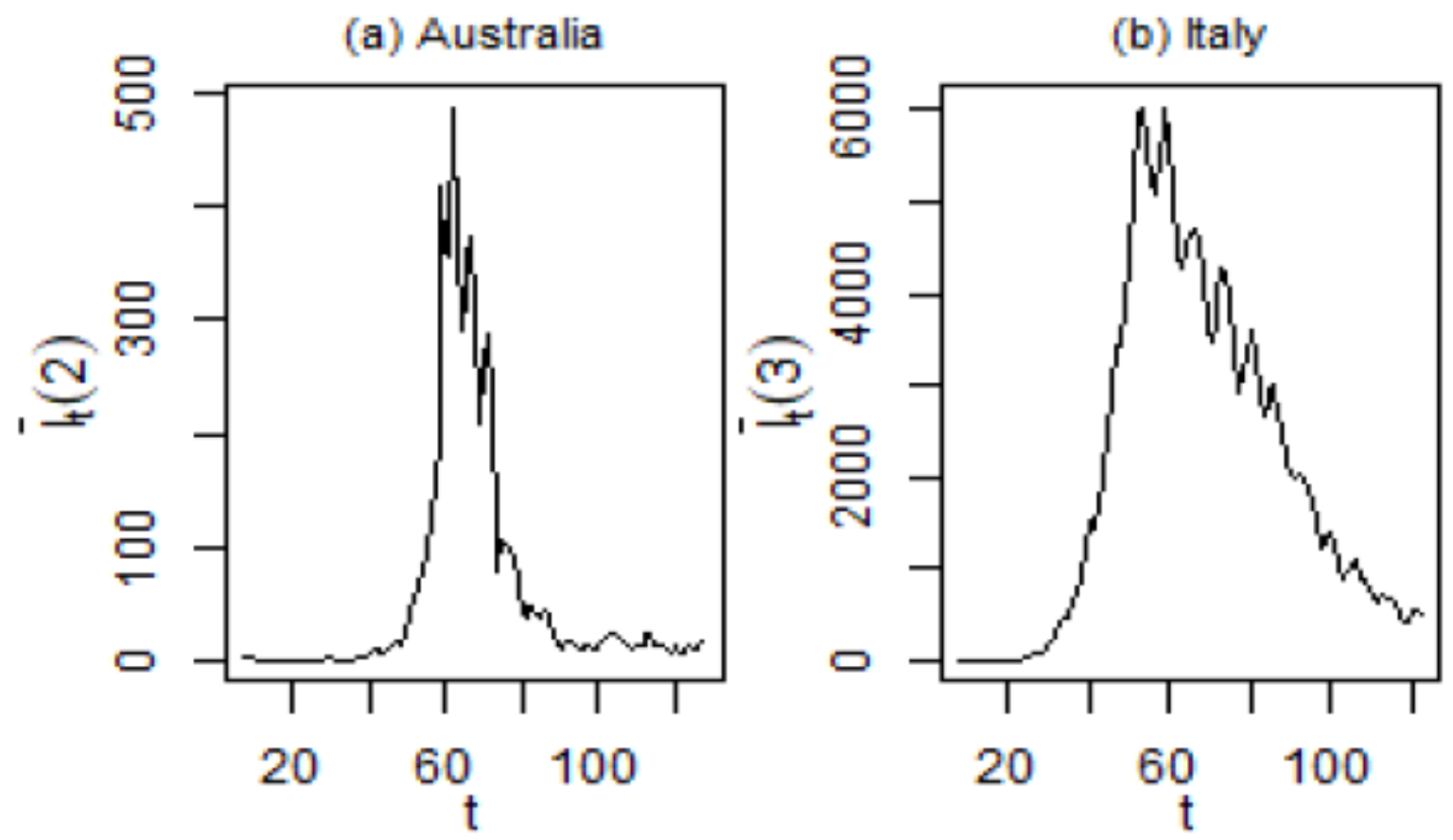

Figure 3

Rolling mean $I^{-} t(\tau)=j=0 I t-j / \tau$ where $t$ is the number of days since the first case 
(a) Density: $50 / \mathrm{km}^{2}$

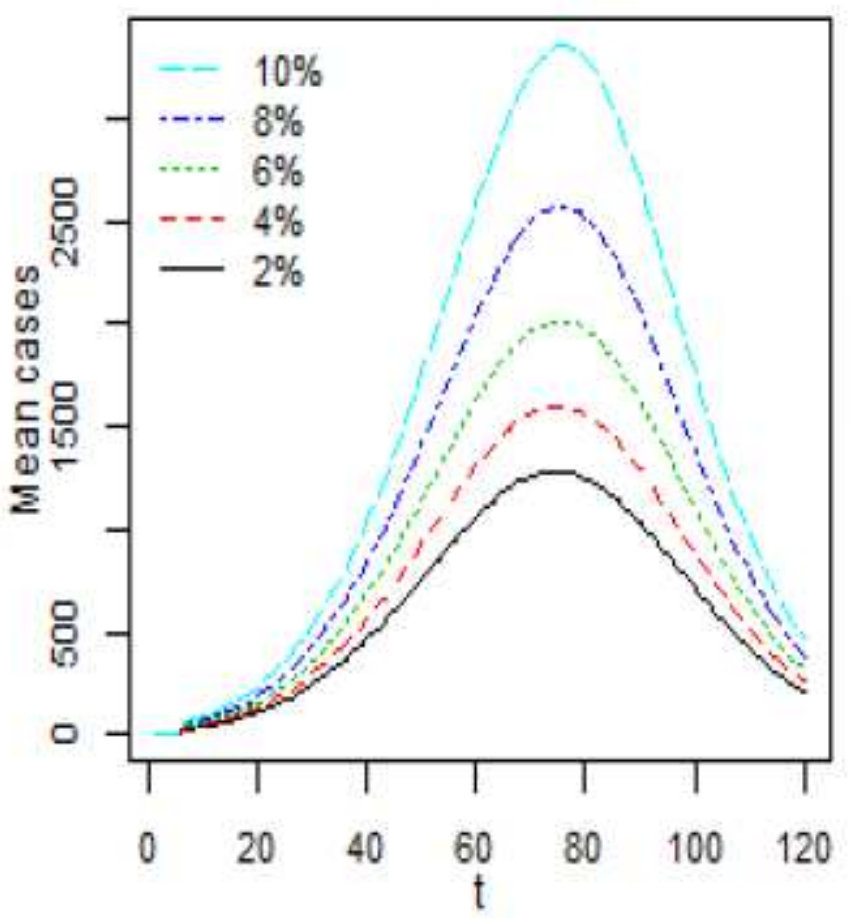

(b) Density: $200 / \mathrm{Km}^{2}$

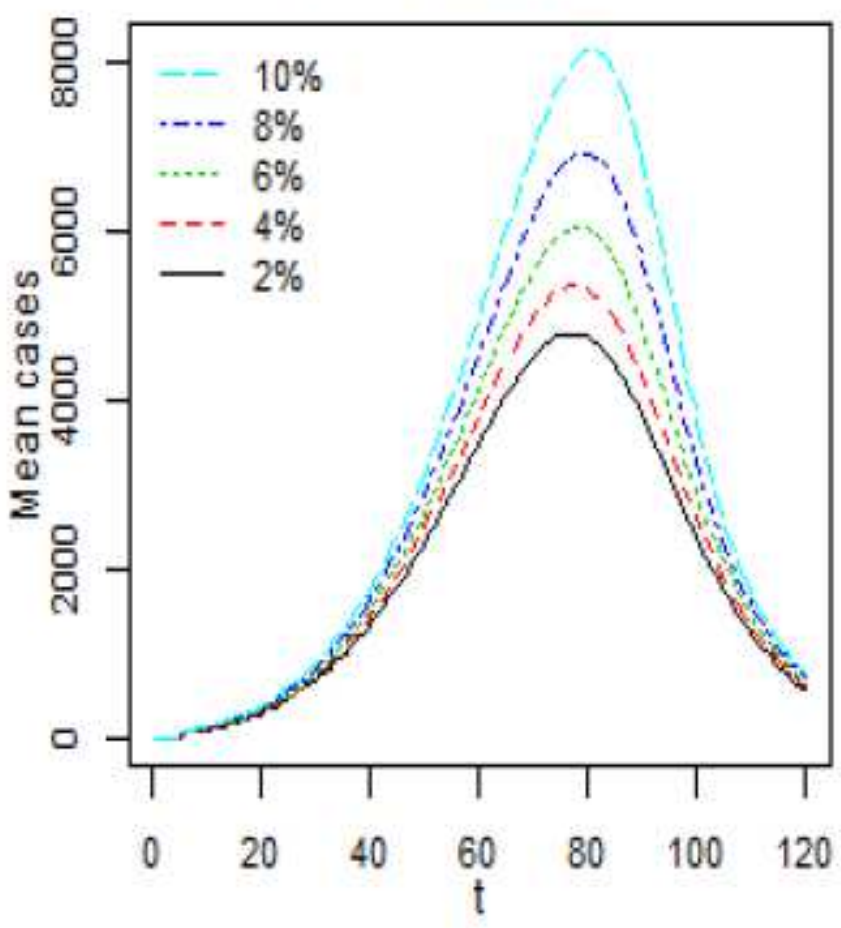

\section{Figure 4}

Distribution of daily number of cases in response to the combined effect of $\log$ (Density) and 5-day lag of test positivity rate $\mathrm{rt}-5$.

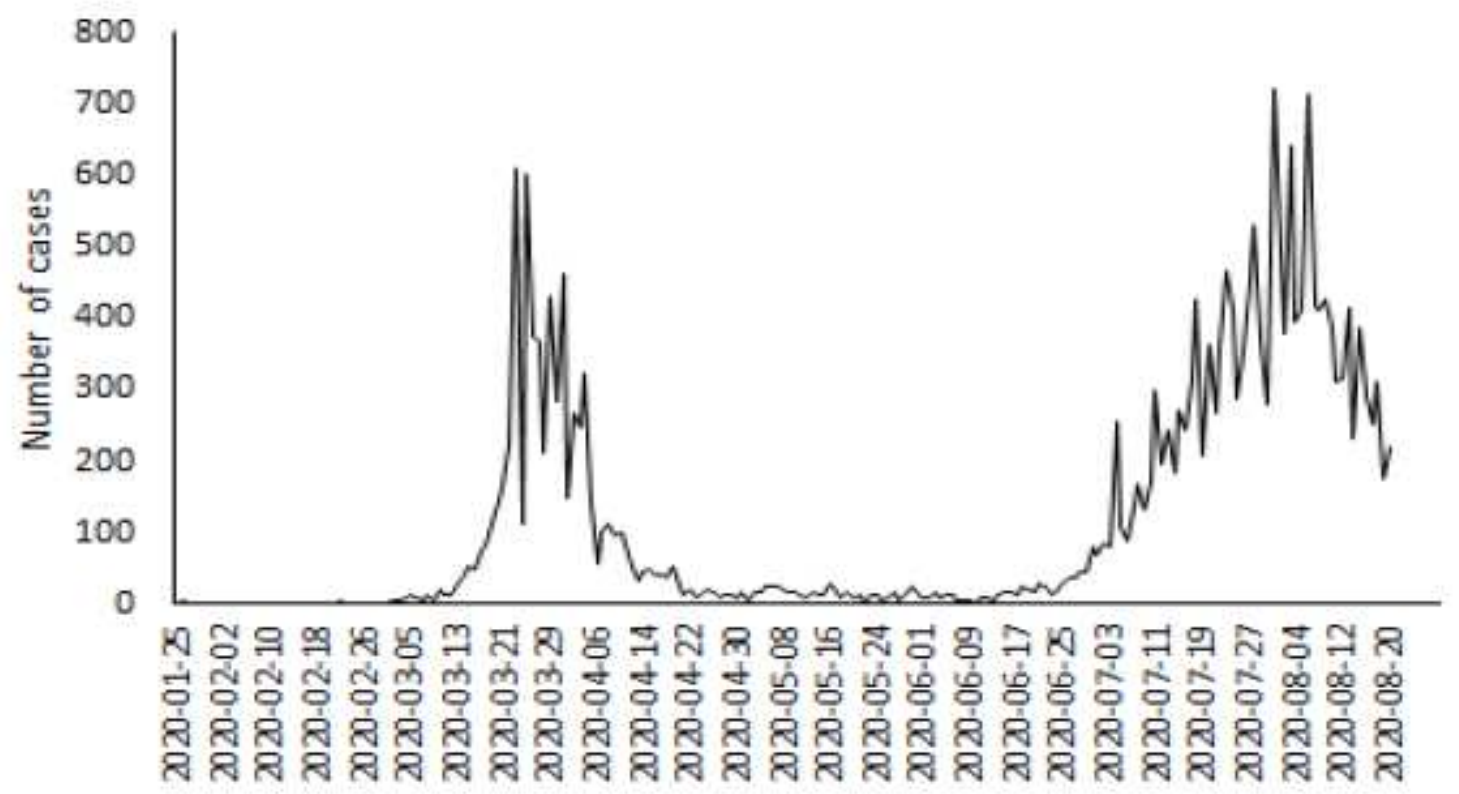

Figure 5

Distribution of daily number of cases during the first and second waves in Australia. 